\title{
Improving doctoral support through group supervision: analysing face-to-face and technology-mediated strategies for nurturing and sustaining scholarship
}

\begin{abstract}
The challenges of the doctoral journey can create social and academic isolation. Student support is normally facilitated through the supervisory team and research training programmes. There is little empirical evidence on the role group supervision and peer learning can play in nurturing and sustaining doctoral scholarship. This article explores group supervision processes, analysing student experiences of face-to-face and technology-mediated $(\mathrm{Tm})$ strategies in a professional doctorate programme, to address the question of what factors in group supervision help or hinder scholarship. Findings illustrate how group supervision can nurture mutual and sustained support and how Tm encounters can add value, affording location-independent interactions to facilitate participation, and reduce isolation. Key dimensions of a pedagogical support framework for doctoral supervision will be identified, which give priority to nurturing relationship development and sustaining connectedness through group supervision. This form of nourished scholarship can support and sustain the doctoral journey and improve completion rates.
\end{abstract}

\section{Keywords}

Doctoral supervision; group support; peer learning; computer-supported learning; well-being

\section{Introduction}

Doctoral education is tasked with contributing to the knowledge economy and performative culture of modern society (Lyotard 1984; Delamont, Atkinson, and Parry 1997; Usher 2002; Barnacle and Dall'Alba 2011). Doctoral students are expected to develop generic research skills to fit them for contributing to 'innovation, entrepreneurship, management and leadership' (Leitch Review 2006) and to demonstrate impact and knowledge transfer through their research (Warry Report 2006). Doctoral education is characterised by increasing provision of nontraditional routes and growth of professional doctorates in a wide range of disciplines geared to developing 'researching professionals' rather than 'professional researchers' (Gregory 1995; Doncaster and Thorne 2000; Bourner, Bowden, and Laing 2001; Usher 2002; Galvin and Carr 2003). The development of these programmes has generated 'creative tensions' (Malfoy 2005) and 'issues of validity' (Costley and Lester 2012) associated with their scope and delineation, particularly when compared with the conventional $\mathrm{PhD}$. These tensions impact on students and supervisor relationships, highlighting the need to consider alternative, more innovative and enabling pedagogic support strategies that can address these challenges and create opportunities for more collective and collaborative research cultures and environments (Delamont, Atkinson, and Parry 1997; Cousin and Deepwell 2005; Malfroy 2005; Samara 2006; Wisker, Robinson, and Shacham 2007) focused on 'nourished scholarship' (Carr, Galvin, and Todres 2010).

Professional doctorate students can experience particular challenges associated with being professionally experienced, mature and part-time students while sharing with all doctoral students the essentially personal and individualised nature of the doctoral experience, routed in the goal of undertaking and successfully completing a sustained in-depth investigation of a problem or issue in order to make a significant contribution to knowledge. This experience can

Reference this paper as: Hutchings, M. (2015). Improving doctoral support through group supervision: analyzing face-to-face and technology-mediated strategies for nurturing and sustaining scholarship. Studies in Higher Education, 2015 http://dx.doi.org/10.1080/03075079.2015.1058352 
create feelings of social and academic isolation, identified as a major contributory factor in doctoral attrition (Delamont, Atkinson, and Parry 1997; Bourner, Bowden, and Laing 2001; Ali and Kohun 2006, 2007). Individual students must negotiate their pathway towards a research degree within this complexity of influences and expectations. While it is thought that group supervision strategies can offer a particular form of support which can nurture and sustain doctoral scholarship and contribute to the reduction in dropout rates, there is little empirical evidence. A pilot research project, to identify how online group supervision could contribute to supporting established peer group supervision within a professional doctorate programme, provided the focus to explore the question of what it is about group supervision and group processes that can support and sustain the students on their doctoral journey and how technology mediated $(\mathrm{Tm})$ group strategies can contribute to nurturing and sustaining scholarship.

Previous work concerning the nature and context of professional doctorates, the kinds of students they attract, the issues and tensions this generates, and the different kinds of pedagogical support strategies required, will be drawn on to inform and ground the research on group supervision before examining the effects of face-to-face (FtF) and Tm strategies through interviews and observation of student experiences. The aim is to identify the key dimensions of a pedagogical support framework for nurturing relationship development and sustaining connectedness during the doctoral student's journey derived from the research findings and informed by the literature on research supervision towards the enhancement of doctoral scholarship and improving doctoral completion rates.

\section{Background}

\section{Nature of professional doctorates and student characteristics}

Professional doctorates have a long academic history, originating in theology, law, and medicine. Provision expanded rapidly in the late twentieth century with professional doctorates in for example, Education (EdD), Business Administration (DBA), and Engineering (EngD) offering alternatives to the Doctor of Philosophy $(\mathrm{PhD})$ route. While professional doctorates can be distinguished from $\mathrm{PhDs}$ by the named professional qualification awarded, it is much harder to distinguish features of 'professional doctorateness' due to marked variations in provision between different universities, disciplines, and even within the same subject domain (Bourner, Bowden, and Laing 2001). Professional doctorates can, however, be characterised by their purpose and design for 'researching or scholarly professionals', who generate new knowledge to inform improvements in practice and 'prepare for higher level professional practice and leadership' rather than 'professional researchers' or 'professional scholars', who generate new knowledge towards scholarly or research activities (Doncaster and Thorne 2000; Bourner, Bowden, and Laing 2001; Galvin and Carr 2003, 294; Costley and Lester 2010).

While the goal of doctoral students, whether as a scholarly professional or professional scholar, is essentially the same, to undertake and successfully complete their doctoral journey, doctoral programmes differ in the kinds of students they attract. The professional doctorate is oriented 
towards experienced practitioners working within different professions. The student profile is one of mature and diverse students juggling commitments to their professions and private lives (Malfroy 2005). The preferred mode of study tends to be part-time (Bourner, Bowden, and Laing 2001; Carr and Galvin 2005), with exceptions like the EngD designed as a high-status route for young engineering graduates studying full-time (UKCGE 2002; Scott et al. 2004). The implications of this student profile for targeted and responsive support are considerable. A UK survey of completion rates demonstrates that part-time students are less likely to complete a PhD than full time students (HEFCE 2007, 14). Part-time study also involves a much higher proportion of older students $(71 \%)$ with statistics showing a negative association between age and rates of PhD completion (HEFCE 2007, 20-21). Boud and Tennant $(2006,299)$ point out the challenges of working with older professionally experienced students where sustaining motivation will 'make substantial inroads into their lives during a period when work and family are of particular significance'. These circumstances combine to make the professional doctorate journey more challenging and isolating for mature part-time students when compared with younger full-time doctoral students.

When these student characteristics are factored in with the diverse nature of professional doctorates, the work of conceptualising and enacting its constituents adds a further dimension to the tensions and issues associated with individuals negotiating their way through it. Where Costley and Lester (2010) focus on the evolution of professional doctorates into a third generation, they characterise these 'work-based doctorates' as transdisciplinary, candidatecentred, research and development programmes negotiated and directed by the candidates themselves within the sphere of work-based learning. This demonstrates the tension for students and supervisors alike in negotiating the flexibility and choice engendered by such programmes while at the same time meeting the rigour and expectations of a doctoral-level qualification situated within university procedures and practices more attuned to working with the conventional $\mathrm{PhD}$ route.

\section{Supervisor-supervisee relationships}

While the perceived tension 'between the need to guide and structure doctoral work on the one hand, and the desire to preserve the doctoral student's autonomy on the other' (Delamont, Parry, and Atkinson 1998, 170) has long been recognised as a tension in the supervisory relationship within all kinds of discipline-based doctorates, negotiating the less familiar territory and constituents of the professional doctorate adds another layer of complexity, contributing to the challenges for the supervisor-supervisee relationship. Malfroy $(2005,171)$ suggests that this issue can be viewed positively as a 'creative tension', 'almost a prerequisite in the process of producing a scholar', but recognises feelings of discomfort and vulnerability expressed by students embarking on doctoral programmes which explicitly link research to workplace practices, and noted student awareness of unequal power relationships with their supervisors.

The experience of power relationships within professional doctorates may be explained by the tensions and challenges to 'expertise' residing on the borders between the different territories 
of academia and workplace and emanating from transitions and transactions between the two domains. On the one side, the experience of transitions from the workplace to academia challenges the 'expertise' of established professionals as they enter the less familiar territory of doctoral research and academic study. On the other side, the encounter is with academics experienced in the research culture of the conventional $\mathrm{PhD}$, familiar with the studentsupervisor relationship, represented in the master-apprenticeship model, where novice students work under the tutelage of an expert supervisor as they prepare for initiation into an academic career, and perhaps less familiar with the domain of the professional doctorate. Gregory (1995, 181) identified a profile of EdD students as senior managers and professionals who 'bring with them their own expertise - in many cases significantly greater (in terms of practice and leadership)' than that of the academics who will be supervising their research. While offering these tensions as an explanation for how encounters can play out, it is possible to argue for a third way by developing 'more equal' supervisor-supervisee relationships, through 'working side by side' (Malfroy 2005, 169). Halse and Malfroy (2010) recognise such a 'learning alliance', but point out that in practice, the relationships are neither equal nor democratic and suggest that this is influenced by the intensification of university pressures on academics steering supervisors to a more disciplined and structured use of time with students focused on meeting milestones and deliverables towards timely completions.

\section{Social isolation and different forms of pedagogical support}

The tensions associated with conceptualising the nature and delineation of the professional doctorate, together with the social and academic impacts for students transitioning from the workplace to academia, where they can feel like a novice, all have potential to contribute to their sense of well-being as they embark on and pursue their doctoral journey. Social and academic isolation is recognised as a specific issue experienced by doctoral students in general, and particularly those embarked on part-time study or working at a distance (Bourner, Bowden, and Laing 2001; Samara 2006; Wisker, Robinson, and Shacham 2007). Isolation has been identified as a major contributory factor in the decision of doctoral students to leave prior to completion (Ali and Kohun 2006, 2007). However isolation should not be conceived simply as a personal construct played out in the life circumstances of individual doctoral students, for it is also associated with the research environment into which doctoral students enter. Delamont, Atkinson, and Parry (1997) observed how the personal and individualised nature of the doctoral experience approximated to social isolation within a critique of 'critical mass' (the numbers of research active individuals considered a necessary and sufficient indicator for creating a research environment for effective doctoral study) and argued that such policy initiatives were insensitive to fundamental differences between different academic disciplines and their modes of organisation. The critical argument here is assuring sequential and pedagogical continuity over critical mass and the value of promoting more collective and collaborative research and learning environments, which do not rely solely on the supervisorsupervisee relationship.

This has important implications for developing an alternative enabling pedagogical framework for nurturing and sustaining scholarship through identifying the different dimensions of 
pedagogical support needed to meet the diverse needs of individual students working in different research environments. It foregrounds possibilities for developing more flexible group forms of support to complement supervisory teams and research training programmes. While the role of the supervisor is not the main focus here, it is important to identify the key components of this 'support' relationship to inform and ground the research focus on group supervision. Lee (2008) recognised the value of supervisory functions, but emphasised the importance of engaging with a conceptual approach towards research supervision, to encompass five concepts: (1) 'functional' duties, (2) 'enculturation', (3)'critical thinking', (4) 'emancipation', and (5) 'relationship development'. These concepts are explained in more detail in Table 1 and provide some of the key dimensions anticipated in a pedagogical support framework for nurturing and sustaining doctoral scholarship. While Lee's (2008) framework is focused on the individual supervisor-supervisee relationship, it acts as a valuable benchmark against which to compare what happens in group supervision. The supervisor role has been associated with different nomenclature and changing relationships; the 'learning consultant' (Boud and Tennant 2006) or the 'guardian supervisor' (Wisker, Robinson, and Shacham 2007), providing one among a number of layers of support, and presaging more equal relationships to address isolation, diversity and distance among doctoral students.

The other major aspect of support is represented by collective and collaborative group forms including research seminar systems (Malfroy 2005), research development workshops, selfhelp groups, symposia (Wisker, Robinson, and Shacham 2007), and group (Samara 2006) or cohort supervision as a resource for 'nourished scholarship' (Carr, Galvin, and Todres 2010). These group approaches, widely practised in undergraduate education (Jaques and Salmon 2007), align with social-constructivist theories of learning, and relate to growing interests in the concept and application of communities of practice and network learning (Cousin and Deepwell 2005; Wisker, Robinson, and Shacham 2007). Professional doctorates are well placed to capitalise on opportunities for providing structured support through group supervision and promoting peer group networking and collaboration when students are recruited to cohorts (Bourner, Bowden, and Laing 2001).

\section{Group supervision}

Group supervision has been widely utilised for clinical supervision in psychology, counselling, and social work practicums, but the research evidence concerning its social and learning benefits for doctoral students is more limited (Enyedy et al. 2003; Linton and Hedstrom 2006; Carter et al. 2009; Fleming et al. 2010; Fenge 2012). Taylor and Beasley (2005) suggest that group supervision offers economic and social benefits, providing a sustainable solution for managing rising numbers of students within increasing academic workloads. The supervisor can save time, giving information to the group rather than individuals, and sharing some supervisory roles with the peer group. Students can provide social and academic support for each other by "sharing research materials, working their way through "blocks" in their research projects, collectively problem-solving, and meeting socially to encourage each other to keep going' (Taylor and Beasley 2005, 96). 
Table 1. Dimensions of pedagogical support for nurturing and sustaining doctoral scholarship

\begin{tabular}{|c|c|c|c|}
\hline $\begin{array}{l}\text { Research supervision concepts } \\
\text { (Lee 2008) }\end{array}$ & $\begin{array}{l}\text { Helping clusters } \\
\text { (Carter et al. 2009) }\end{array}$ & $\begin{array}{l}\text { Hindering factors } \\
\text { (Enyedy et al. 2003) }\end{array}$ & $\begin{array}{l}\text { Nourished scholarship dimensions } \\
\text { (Carr, Galvin, and Todres 2010) }\end{array}$ \\
\hline $\begin{array}{l}\text { (1) Functional } \\
\text { Directing, project management, } \\
\text { progress checking }\end{array}$ & $\begin{array}{l}\text { (1) Specific instruction } \\
\text { Objective knowledge, ethical issues, } \\
\text { disciplinary and professional information }\end{array}$ & $\begin{array}{l}\text { (1) Logistical constraints } \\
\text { Room size, inappropriate time of day }\end{array}$ & $\begin{array}{l}\text { (1) Negotiating the rhythms of receptive and } \\
\text { active times } \\
\text { Working with a needs-led agenda, flexible and } \\
\text { negotiable around the force of time for } \\
\text { stepping back and stepping forward }\end{array}$ \\
\hline $\begin{array}{l}\text { (2) Enculturation } \\
\text { Initiating and developing the } \\
\text { student as a member of the } \\
\text { disciplinary community }\end{array}$ & $\begin{array}{l}\text { (2) Peer impact } \\
\text { Diversity and multiplicity of views, learning } \\
\text { from each other, peer feedback, learning } \\
\text { vicariously through others' experiences, } \\
\text { collaborative problem-solving }\end{array}$ & $\begin{array}{l}\text { (2) Between-member problems } \\
\text { Competitiveness, criticism, collective } \\
\text { griping, non-participation }\end{array}$ & $\begin{array}{l}\text { (2.1) Belonging } \\
\text { Being with others who, while pursuing } \\
\text { different research topics, are on a similar } \\
\text { journey } \\
\text { (2.2) Scholarly community } \\
\text { Relating and being accountable to "a broader } \\
\text { community and its tradition and scholarship" }\end{array}$ \\
\hline $\begin{array}{l}\text { (3) Critical thinking } \\
\text { Challenging the student to } \\
\text { analyse and question their own } \\
\text { work }\end{array}$ & $\begin{array}{l}\text { (3) Supervisor impact } \\
\text { Openness, humour, sharing past experiences, } \\
\text { validating supervisees, individual feedback, } \\
\text { making time for all supervisees' issues }\end{array}$ & $\begin{array}{l}\text { (3) Problems with supervisors } \\
\text { Dominating the group, overly critical, } \\
\text { unfocused, lacking experience }\end{array}$ & $\begin{array}{l}\text { (3) Growing confidence } \\
\text { Where the group is used as a sounding board } \\
\text { for ideas }\end{array}$ \\
\hline $\begin{array}{l}\text { (4) Emancipation } \\
\text { Mentoring to inspire student } \\
\text { personal development and self- } \\
\text { discovery }\end{array}$ & $\begin{array}{l}\text { (4) Self-understanding } \\
\text { Self-knowledge and personal growth }\end{array}$ & $\begin{array}{l}\text { (4) Poor group time management } \\
\text { Supervisees not getting their share of } \\
\text { the allocated time }\end{array}$ & $\begin{array}{l}\text { (4) Values clarification } \\
\text { Enabling "one's own emerging and changing } \\
\text { identity to be articulated "around being and } \\
\text { becoming }\end{array}$ \\
\hline $\begin{array}{l}\text { (5) Relationship development } \\
\text { Emotional intelligence and } \\
\text { flexibility deployed to enthuse, } \\
\text { nurture and care for the } \\
\text { student }\end{array}$ & $\begin{array}{l}\text { (5) Support and safety } \\
\text { Group dynamics, emotional security and } \\
\text { intimacy }\end{array}$ & $\begin{array}{l}\text { (5) Supervisee anxiety } \\
\text { Feeling unsafe, emotionally } \\
\text { unsupported, sensitive to criticism }\end{array}$ & $\begin{array}{l}\text { (5.1) Containing anxiety } \\
\text { Where risks are acknowledged and } \\
\text { humanised } \\
\text { (5.2) Commonality and uniqueness } \\
\text { Welcoming novelty and surprise and learning } \\
\text { from each other through the 'gift of } \\
\text { cohortness' }\end{array}$ \\
\hline
\end{tabular}

Reference this paper as: Hutchings, M. (2015). Improving doctoral support through group supervision: analyzing face-to-face and technology-mediated strategies for nurturing and sustaining scholarship. Studies in Higher Education, 2015 http://dx.doi.org/10.1080/03075079.2015.1058352 
But the social learning benefits of group supervision are dependent on mediating factors at work in group processes. Carter et al. (2009) identified five clusters of helpful events in group supervision: (1) specific instruction, (2) peer impact, (3) supervisor impact, (4) selfunderstanding, and (5) support and safety. Table 1 outlines these helping processes and aligns them with the supervisory concepts identified by Lee (2008). Conversely Enyedy et al. (2003) identified five group processes, which hindered supervisee development in group supervision: (1) logistical constraints, (2) between-member problems, (3) problems with supervisors, (4) poor group time management, and (5) supervisee anxiety. These hindering factors are also linked with the dimensions offered by Lee (2008) and Carter et al. (2009) in Table 1.

What is particularly significant here is the social interactions between participants both supervisors and peers as key sources of impact either helping or hindering group processes, peer learning and relationship development. Carter et al. $(2009,7)$ noted: 'Lack of trust and safety hampered supervisees' ability to share experiences, and resulted in a defensive attitude counterproductive to real self-exploration'. Alternatively, where there was a sense of cohesion, described by Linton and Hedstrom $(2006,58)$ as 'warm and comfortable feelings', participants reported that they were able to provide and receive 'constructive criticism' without becoming 'defensive' and to feel valued and respected for having differing opinions and points of view. Effective facilitation of 'relationship development' appears a fundamental prerequisite for stimulating 'critical thinking' and effecting 'emancipation' whether the context is a direct supervisee-supervisor relationship or group supervision. This notion is reflected in the work of Carr, Galvin, and Todres (2010), who highlighted the emotionally complex experience of the doctoral journey, identifying 'cohort supervision' as the primary resource for facilitating the phenomenon of 'nourished scholarship'. They provide a framework for focusing attention on the importance of the emotional journey and how to sustain students' motivation and resilience through consideration of seven dimensions defined in Table 1: (1) negotiating the rhythms of receptive and active times, (2.1) 'belonging', (2.2) 'scholarly community', (3) 'growing confidence', (4) 'values clarification', (5.1) 'containing anxiety', and (5.2) 'commonality and uniqueness' (Carr, Galvin, and Todres 2010).

\section{Tm communication}

The facilitation of group supervision through $\mathrm{Tm}$ communication (TmC) adds a further dimension, which may help or hinder relationship development and 'connectedness' for nourished scholarship. Very little research appears directly related to the effects of $\mathrm{TmC}$ in group supervision. Cummings explored the use of text-based chat in counselling supervision identifying the 'disinhibition' effect of online communication in the absence of FtF contact. He reported that text-based communication supported open and honest communication and enabled feelings to be communicated in this space 'with surprising ease' (Cummings 2002, 223).

The literature on computer-mediated communication emphasises the value of collaborative approaches to learning associated with the networked learning movement (McConnell 2000; 
Steeples and Jones 2002; Goodyear et al. 2005) and increasingly promoted through Web 2.0 and social networking. Goodyear et al. $(2005,474)$ argue that 'use of online materials is not a sufficient characteristic to define networked learning' because 'human-human interaction' is essential. Steeples and Jones' (2002) definition of networked learning focuses on connections between learners, between learners and their tutors, and between a learning community and its learning resources, recognising the importance of 'connectedness' associated with the peer group, nature of collaboration and online presence.

\section{Methodology}

Online group supervision was introduced as a pilot project to support established group supervision for three cohorts within a professional doctorate programme at a UK university in 2011. The purpose of the professional doctorate is to enable experienced professionals engaged in, or affiliated to health and social care, to undertake an open pathway of study, conducting an inquiry into a subject of their own choice, comprised of four elements which constitute the thesis: a research investigation, a practice development project, a literature review, and personal narrative. The professional doctorate cohorts attract different mixes of professions each year including (a) nurses and midwives, (b) physiotherapists, occupational therapists, and paramedics, (c) social workers, and (d) higher education lecturers. Cohorts consist of between four and six students who meet monthly throughout the year for group supervision with two academic group supervisors, whose role in facilitating the groups runs in parallel to that of the student's primary supervisors. The three cohorts recruited were at different stages of their professional doctorate journey, Cohort A commenced in 2008 (four students constituted from professional groups a, b and d)), Cohort B in 2009 (four students from a, c, and d), and Cohort C in 2010 (six students from a, c, and d). Students and supervisors were invited to participate in online group supervision using the synchronous features of a virtual learning environment (VLE), including video-conferencing, text communication via online chat, and content display for sharing web pages and documents via a whiteboard. Participants were invited to take part in two online sessions: first, an induction, providing opportunities to try out sharing documents and websites and to communicate online, using video-conferencing and text chat; second, an online group supervision meeting, to enable participants to experience online group supervision working remotely from their workplace or home. Participants were encouraged to test devices and connections so that technical issues could be dealt with prior to participation in the live sessions.

The research methodology adopted was qualitative with the aim of recording students' and supervisors' experiences of group supervision, both $\mathrm{FtF}$ and online. The methods involved participant observation of live group supervision sessions, semi-structured group interviews conducted at the end of online sessions, and an open-response questionnaire sent to students and supervisors to capture reflections following the online sessions. The technology enabled the supervisory process to be directly observed and interactions were recorded and archived through audio, video and text chat channels. Ethical issues were addressed by seeking relevant permissions and peer review for the student informed consent process with a participant 
information sheet, written consent, and an independent contact for participants with any concerns.

\section{Findings}

The purpose of the research was to address two key questions:

What is it about the group process and group supervision that supports and sustains the doctoral journey?

How can technology-mediated encounters contribute to group process in supporting and sustaining the doctoral journey?

Student comments are linked to their respective cohort by letters A, B and C and supervisor comments are distinguished by an S. The analysis shows how roles traditionally associated with the supervisor are facilitated through the peer group.

\section{Helping factors in group supervision}

Functional: project management and progress checking to knowledge sharing

Students recognised functional benefits (Lee 2008) of group supervision, 'to encourage moving forward with tasks' (A), including sharing knowledge on transcribing interviews, data analysis, and remaining 'focused within the research project' (C).

Sharing of information is really helpful, for example, the systematic literature review. Gems like that create

light bulb moments and give inspiration to move forward. (A)

While associated with the 'specific instruction' cluster of Carter et al. (2009), it is not confined to the expertise of supervisors. Students identified various benefits of presenting their work and having discussions at group supervision sessions, for 'exchange of knowledge between colleagues' (A), 'to share ideas that will contribute to each other's research' (C) and to 'bounce ideas from peers' (A).

Enculturation: recognising differences while developing as a member of a scholarly community The process of enculturation (Lee 2008) relates to becoming a scholarly professional. Students recognised differences through 'sharing the different professional perspectives' (C) and they also described 'going through similar things' (C) and identified the value of group supervision 'to get support from like-minded individuals' (B). They described how getting together could help with feelings of isolation on the doctoral journey.

Regular meetings - you look forward to them and you don't feel so isolated in your work. (A)

Getting together reminds you, you are 'part' of something as doctoral studies can be a lonely journey. (B)

\section{Critical thinking: challenging own work}

The link between critical thinking and relationship development (Lee 2008) was identified when students described the purpose of group supervision 'to learn to debate and justify

Reference this paper as: Hutchings, M. (2015). Improving doctoral support through group supervision: analyzing face-to-face and technology-mediated strategies for nurturing and sustaining scholarship. Studies in Higher Education, 2015 http://dx.doi.org/10.1080/03075079.2015.1058352 
pathways chosen in a safe environment' (A). The 'support and safety' built around group dynamics, emotional security and intimacy (Carter et al. 2009) appeared to be a major influence in promoting critical thinking as students recognised how group supervision can 'stimulate debate and ideas' (B) and provide 'a safe environment to defend your research' (A).

\section{Emancipation: inspiring personal development and self-discovery}

The frequency of meetings and the opportunity to gauge oneself against one's peer group were recognised as important motivational facets supporting 'personal development and selfdiscovery' (Lee 2008):

The opportunity to reflect on one's progress with other people. It enables me to measure myself against others. It gives me the added drive to do something. (A)

\section{Relationship development: emotional intelligence and flexibility}

Students noted benefits of group supervision in providing 'ongoing support and encouragement' (B) and the value of 'getting to know and trust' (B) the group over time:

It offers sympathy and support in difficult situations - ups and downs on the DProf journey. (A)

We know we are there for each other and whatever we say stays within our group. (C)

\section{Hindering factors in group supervision}

While Enyedy et al.'s (2003) five processes hindering supervisee development were not specifically identified, students recognised one of the 'between-member problems', nonparticipation, as a factor hindering the group supervision process. Comments included 'poor attendance' (B) and 'others not engaging' (B), but students recognised this was associated with difficulties in managing time in their busy professional lives:

Time is the biggest thing, it's often difficult to get even the two hours required for the group supervision sessions. (A)

The question of structure and flexibility in group supervision was identified, where it was described as 'unfocused' (B) with lack of preparation and planning (B). Comments related to different expectations of what group supervision should be (BS) and highlighted the balance between structure and flexibility as a theme for further discussion.

\section{Helping factors in Tm group supervision}

Participants felt that technology would help overcome hindering factors in group supervision like managing time and availability for meetings: 'Juggling work life, things like this could make it easier' (A). The facility for location-independent interactions through remotely accessing the shared space for online group supervision was welcomed by students and supervisors: 
I do 25,000 miles a year - so anything that helps me reduce miles / save time and still allows me to think and talk is great. (A)

It would overcome issues of people needing to come from all over to one location. (BS)

Participants identified the most helpful features in the VLE, ranking document sharing as the most popular, followed by remote access and sharing web resources, then synchronous dialogue. While some participants felt self-conscious about seeing themselves through the live video links when speaking, they felt that they 'would get used to it' (C). Fewer participants selected instant messaging as helpful and the emoticons were least popular. Participants' familiarity and confidence with the technology and interacting online developed over the two sessions: 'The second time - getting easier. If we had a monthly session, we would use more effectively' (AS).

\section{Hindering factors in Tm group supervision}

Hindering factors fell into two categories: extra-technology associated with technical-systemic factors external to, but impacting on the online experience; and intra-technology factors associated with the VLE's features.

\section{Extra-technology factors}

Technical difficulties were encountered by students and supervisors, some of which could not be resolved in advance of the online sessions. Issues included strength of network connections, slow internet speeds, intermittent connectivity, computer security blocking software, and difficulties experienced when switching between different device configurations in different locations. The motivational impacts and resource implications of these extra-technology factors were summarised in comments like:

It does require a greater understanding of IT issues - but if I use it regularly I am sure that these can be overcome. (A)

Makes assumption everyone has access to required and correctly configured equipment and is IT literate, this will discourage people from engaging. (B)

\section{Intra-technology factors}

Group interactions were affected by the software configuration which only enabled the speaker to be seen. As a result, participants felt group interaction was more limited online:

Not seeing everyone at the same time took away some of the body language that you would normally see in a group. (A)

The interpersonal cues and prompts in FtF group supervision were missed in online meetings. People were talking over each other through the audio feed, and while some participants liked the 'ability to write at the same time' (C), others were distracted by instant messaging which encouraged 'a kind of sub conversation' (C). Participants felt that online conversations lacked 
spontaneity and failed to flow. This raises questions about the nature of communicative interactions and sense of connectedness for online group supervision.

\section{Discussion}

Four key themes contributing to doctoral support emerged from the findings: the value of regular peer group communication and connectedness for developing a sense of belonging; the importance of negotiating the balance between structure and flexibility for containing anxiety and building confidence towards emancipation; factoring in more time for reducing the impacts of systemic and technical factors to ensure that motivation is not compromised; and challenging the prevailing culture of individual, $\mathrm{FtF}$ supervision, to inform the nature and processes of student support for facilitating online as well as FtF group supervision.

\section{Regular communication and connectedness for developing a sense of 'belonging'}

The value of group supervision, in bringing together 'like-minded individuals', is central to the process of enculturation and reflected in 'belonging' one of the key dimensions of 'nourished scholarship' identified by Carr, Galvin, and Todres (2010). The findings demonstrate the significance of relationship development for building 'a sense of shared values' (C) associated with experiencing a similar journey towards becoming a scholarly professional. The significance of relationship development for nurturing connectedness is expressed in comments like:

The group supervision has at times been the only thing keeping me going. (A)

It helps to feel connected to a group. It can be quite isolating without it. (C)

Participants recognised that the technology could 'improve the student experience as it was a way to communicate with individuals if a face-to-face meeting wasn't possible' (A). But the sense of connectedness developed by coming together regularly with a common purpose is strong and it is perhaps not surprising that participants, both students and supervisors, expressed some doubts about how using TmC could possibly compare with the strength of support in FtF group supervision:

The strength of the supervision session is the 'face-to-faceness' of the support received. Technology takes this away. (BS)

Group C students, in online dialogue, suggested that it was not possible to generate the same degree of presence and connectedness online when compared to FtF meetings:

'The personal feeling is lost a bit'. 'It does feel a bit more distant. 'We haven't said as much as normal'.

Walther's (1996) experimental study on impersonal, interpersonal and hyperpersonal interaction suggests that these reactions can be explained by considering combinations of media attributes, social phenomena, and social-psychological processes associated with interpersonal communication facilitated through different media. The range of media channels 
available affects the degree of attention that can be paid by the participant to the presence of other participants in an interaction. For example, text-based communication can be described as 'lean' while audio-video channels offer 'rich media'. However, this does not mean that, as cues are filtered out, social presence necessarily declines with messages becoming more impersonal or disclosure and intimacy diminishing. In fact, Tidwell and Walther (2002) found that people make more intimate self-disclosures in $\mathrm{TmC}$ than $\mathrm{FtF}$ interactions, which may support Cummings 'disinhibition' effect (2002).

What distinguishes social information processing is the rate rather than the amount of social information exchanged, with less information per message in $\mathrm{TmC}$ compared to $\mathrm{FtF}$ due to the absence of non-verbal cues (Walther 1996). While the online supervision experience afforded richer media channels with audio, visual and text for social information exchange, it appeared to constrain participants used to $\mathrm{FtF}$ meetings. Intimacy and self-disclosure are key concepts in relationship development and build on reciprocity between individuals. TmC, like FtF relationships, is dependent on partner responsiveness (Jiang, Bazarova, and Hancock 2011), but additional time is required to facilitate social over task-oriented interactions. When the media rate is factored in with time online, the contribution to relationship development has greater potential to be realised.

This helps to explain some of the initial reactions of participants, unfamiliar with the affordances of $\mathrm{TmC}$, and highlights the importance of allowing more time for online strategies to be embedded in group supervision practice. In fact, observation of the online supervision meetings revealed a surprising amount of openness and willingness to share personal and professional issues and to demonstrate awareness, concern and emotional support, expressed through verbal comments and instant messaging. Although colleagues suggested that it was harder to respond appropriately online compared to $\mathrm{FtF}$, group member responses were very supportive, prompting one student to say: 'The level of disclosure did not seem to be affected by the method of communication' (C).

\section{Negotiating structure and flexibility for containing anxiety and building confidence}

The balance between structure and flexibility emerged as another theme linked to the conduct of group meetings and the nature of relationships fostered $\mathrm{FtF}$ and online. Where students may be attracted to professional doctorates providing more structured and taught components (Bourner, Bowden, and Laing 2001), others offer considerably more flexibility in the negotiation of constituent elements such as the work-based doctorates described by Costley and Lester (2012). This has implications for student and tutor conceptions of the doctoral supervision process and presents challenges for the mix of student-managed and tutor-led activities. It highlights the significance of promoting and maintaining non-hierarchical relationships through encouraging what Fleming et al. (2010) describe as 'fluid leadership' in group supervision interactions whether conducted FtF or online. Both students' and group supervisors' comments reflected this approach:

Everyone plays an equal and active part in community and sharing (B). 
The students lead the sessions and talk about what is important for them (CS).

Yet, the 'quiet 'rumble' of emotions described by Malfroy as pointing to the 'ongoing unease about roles and expectations that occur during doctoral candidature' $(2005,170)$, were also displayed by the students and supervisors experiencing online supervision. When sharing observations on how sessions progressed, participants expressed being tentative and unsure how to interact online and valued the more structured approach of the induction session.

I enjoyed the trial session, learning to use the technology and sharing documents, but when we had the supervisory meeting, it seemed a bit clinical and detached. (C)

The transition into a new learning environment, the novelty and lack of familiarity, is marked by creative tensions where students and supervisors are finding their way and learning how to share, lead and talk in a new and unfamiliar learning space. If a degree of anxiety can be recognised as a normal and integral part of the doctoral research journey, just as it is a necessary part of individual learning (Fleming et al. 2010), then online group processes can be harnessed to enable students to express concerns openly in a supportive environment focused on building trust, and mirroring FtF group supervision interactions. Group interactions enable students to recognise the value of the group supervisor role and their peers in regulating the ebb and flow of these tensions: 'Tutors share so much of their own experience and help us make sense' (C). Relationship development in group supervision, whether conducted FtF or online, is founded on collegiate and participatory approaches, an organic partnership in which peers and group supervisors develop a sense of belonging and connectedness, helping to contain anxiety and build confidence over time.

\section{Recognising the benefits while reducing the impact of technical-systemic factors on the student experience}

The notion of relationship development, building nourished scholarship, is feasible in online group supervision, provided technical-systemic issues can be resolved. Benefits recognised included greater opportunities for 'keeping in contact'; enabling synchronous dialogue outside timetabled FtF sessions; facilitating more informal one-to-ones; and increasing the frequency of meetings particularly at key stages, for example, pre-MPhil transfer, but not at the expense of replacing FtF meetings. However the degree of preparation, testing, and support required to reduce technical difficulties in advance of scheduled online synchronous group sessions cannot be underestimated. The poor experience of the preparatory session by one of the groups had a direct influence on participants' willingness to engage in subsequent sessions: 'Personally it lessened my experience considerably and I would not willingly choose this mode of communication' (BS).

The technical issues identified can be attributed to extra-technology technical, systemic, and human factors. Systemic issues were associated with hardware devices not being fit for purpose, particularly for audio transmission but, with more time devoted to preparation and testing, some of these connection issues could have been resolved. Participants, unable to make 
time to test their devices and network connections prior to online sessions, risked missing parts of scheduled sessions with direct impacts on group members whose attention and time were diverted from the session proper in trying to help their colleagues solve technical problems. The flexibility afforded for working at a distance meant that some participants did not schedule start times to synchronise with other participants logging on to online sessions, which also impacted on the online experience: 'Participants need to be ready at the start - the half hour wait could alter the perception of the tool' (B).

Intra-technology factors associated with the software's affordances were also found to have influenced online experiences. The question of social presence expressed through feelings of being detached or connected may prove less of an issue over time as participants familiarise themselves with the technology. However, early socialisation into online group supervision would benefit from strategies, which encourage an effective blend of structure and flexibility to facilitate socio-emotional as well as practical task-oriented interactions. The issue of ensuring that sufficient time is given to developing familiarity, confidence and comfort in this new space is important. A pertinent observation from one participant suggested that: 'The problem seems to me we are trying to do too much in a short time!' (B). Organisational demands on systems, and support for its implementation and continuation also needs to be recognised: 'Use of such systems makes lots of assumptions about what we want and how we achieve it, and what resources everyone has to engage with it' (B).

\section{Challenging the prevailing culture of one-to-one supervision}

The introduction of online group supervision has revealed challenges for change for individuals and for organisations. It has been both welcomed and resisted by individuals. While Cummings argued the strength of $\mathrm{TmC}$ in group supervision by 'bringing people together in ways which honour and affirm the beauty of the human relationship' (2002, 223), the participants in this research study did not see online supervision as a replacement for FtF interactions, but as a useful adjunct to communication for use in group and one-to-one supervision: 'I really like to be able to have "real time" contact over our usual sessions, but not instead of" (C).

However the analysis of cultural change necessary goes beyond individual reactions and resource implications to consider wider organisational and policy debates. Group supervision is a significant form of support and challenges the narrow concept of doctoral education as 'research training' which Barnacle and Dall'Alba identified as focusing 'on technical skills, rather than the craft or artistry of research required for genuine skilful performance' (2011, 465-466). Similarly, Boud and Tennant argue for moving away from the concept of 'provision' to a "discourse of "peer learning" (2006, 303). The assumptions and constraints under which doctoral education operates need to ensure that social learning benefits of group supervision, $\mathrm{FtF}$ and online are not perceived as a deficit model for students who do not aspire to careers in academia and research and need additional support to develop as 'researching professionals' (Bourner, Bowden, and Laing 2001, 71). 
While not replacing the conventional supervisor-supervisee relationship, group supervision offers an alternative by providing an enabling space for the enactment of the dimensions of 'nourished scholarship' (Carr, Galvin, and Todres 2010), facilitating relationship development and connectedness over time. It is in this sense that group supervision adds value by moving beyond the 'interdependence of knowing and doing' associated with the epistemology of the professional doctorate (Costley and Lester 2012) to recognise 'being', through enabling the doctoral student to be comfortable in the transitional spaces between the workplace and the creative tensions and uncertainties associated with becoming a scholarly professional. As such, the benefits of group supervision and potential of online strategies should be encouraged across all doctoral education. Furthermore, Boud and Tennant's (2006) view of the value of supervisors, supporting transdisciplinary work and cooperation required to facilitate new forms of doctoral education has resonance with the argument for developing a particular kind of supervisor able to facilitate group supervision in sympathetic and equal ways, not constrained by their discipline, and open to belonging and performing in a transdisciplinary research culture.

Limitations of the study concern the small number of participants in each cohort and the short time frame for the pilot project. While the number of participants was small, reactions were broadly similar across the groups, though Cohort B's experience was atypical and strongly influenced by extra-technology factors. It would have been beneficial to observe and report on student and supervisor experiences over a longer period to identify if more time for familiarisation and working with TmC would increase its acceptability 'as one of a toolbox of communication strategies' (BS) to support doctoral programmes.

\section{Conclusions}

The landscape of doctoral education is changing with the rapid growth of new forms, particularly professional doctorates attracting part-time and professionally experienced students. The pressure to improve completion rates is considerable and professional doctorate students experience particular challenges in negotiating their doctoral journeys. Group supervision processes were explored, analysing student and group supervisor experiences of participating in $\mathrm{FtF}$ and $\mathrm{TmC}$ strategies in a professional doctorate programme, to address the question of what factors help or hinder doctoral scholarship.

Group supervision contributes to engaging with doctoral students and can do much to support students who are feeling academically and socially isolated. Group supervision offers an enabling and complementary support to the conventional supervisor-supervisee relationship and enables the doctoral student to be comfortable in the creative transitional spaces wrought by the challenges and uncertainties associated with becoming a scholarly professional. Group supervision was found to nurture mutual and sustained support with students sharing emotional and practical issues and receiving comments and feedback from the group to support their professional doctorate journey. TmC can add value by affording location-independent interactions where participants can meet online, to increase student participation and reduce 
isolation. TmC can enhance group supervision by affording greater flexibility and convenience for those unable to attend physically. However achieving an effective balance between structure and flexibility with a blend of socio-emotional and task-oriented-practical interactions is dependent on factoring in cultural considerations and media channel time, particularly for TmC, to deal with novelty and unfamiliarity, and with technical-systemic considerations. Technology issues with hardware and software configurations and network performance are not insubstantial and will discourage people from engaging where insufficient attention is given to providing software and hardware fit for purpose.

The key dimensions in a pedagogical support framework for doctoral supervision, which give priority to nurturing relationship development and sustaining connectedness, have been identified. This form of nourished scholarship realised through group supervision can support and sustain the doctoral journey and improve completion rates. Realising the benefits of nourished scholarship through group supervision takes time, resources, and commitment to embed, particularly when introducing and building familiarity with novel forms of TmC. Technology has an important part to play in enhancing group supervision within the changing research culture and environment of higher education, but will not easily replace the strong 'sense of emotional togetherness', the 'gift of cohortness', developed through FtF group supervision.

\section{References}

Ali, A., and F. Kohun. 2006. Dealing with isolation feelings at IS doctoral programs. International Journal of Doctoral Studies, 1: 21-33.

Ali, A. and F. Kohun. 2007. Dealing with social isolation to minimize doctoral attrition: a four stage framework. International Journal of Doctoral Studies 2: 33-49.

Barnacle, R., and G. Dall'Alba. 2011. Research degrees as professional education? Studies in Higher Education 36 (4) 459-470.

Boud, D., and M. Tennant. 2006. Putting doctoral education to work: challenges to academic practice. Higher Education Research \& Development 25(3) 293-306.

Bourner, T., R. Bowden, and S. Laing. 2001. Professional doctorates in England. Studies in Higher Education 26 (1): 65-83.

Carr, E., and K. Galvin. 2005. Doctoral preparation: issues and relevance for clinical leaders. Journal of Research in Nursing 10 (6) 601-624.

Carr, E., K. Galvin and L. Todres. 2010. Facilitating nourished scholarship through cohort supervision in a professional doctorate programme. Encyclopaideia 14 (27) 125-146.

Carter, J.W., K. Enyedy, R.K. Goodyear, F. Arcinue, and N. Puri. 2009. Concept mapping of the events supervisees find helpful in group supervision. Training and Education in Professional Psychology 3 (1) $1-9$.

Costley, C., and S. Lester. 2012. Work-based doctorates: professional extension at the highest levels. Studies in Higher Education 37 (3) 257-269.

Cousin, G., and F. Deepwell. 2005. Designs for network learning: a communities of practice perspective. Studies in Higher Education 30 (1) 57-66.

Cummings, P. 2002. Cybervision: virtual peer group counselling supervision - hindrance or help? Counselling and Psychotherapy Research 2 (4) 223-229. 
Delamont, S., P. Atkinson, and O. Parry. 1997. Critical mass and doctoral research: reflections on the Harris Report. Studies in Higher Education 22 (3) 319-331.

Delamont, S., O. Parry and P. Atkinson, and. 1998. Creating a delicate balance: the doctoral supervisor's dilemmas. Teaching in Higher Education 3 (2) 157-172.

Doncaster, K., and L. Thorne. 2000. Reflection and planning: essential elements of professional doctorates. Reflective Practice 1(3) 391-399.

Enyedy, K., F. Arcinue, N. Puri, J.W. Carter, R.K. Goodyear, and M. Getzelman. 2003. Hindering phenomena in group supervision: implications for practice. Professional Psychology: Research and Practice 34 (3) 312-317.

Fenge, L-A. 2012. Enhancing the doctoral journey: the role of group supervision in supporting collaborative learning and creativity. Studies in Higher Education 37 (4) 401-414.

Fleming, L., J. Glass, S. Fujisaki, and S. Toner. 2010. Group process and learning: a grounded theory model of group supervision. Training and Education in Professional Psychology 4 (3) 194-203.

Galvin, K., and E. Carr. 2003. The emergence of professional doctorates in nursing in the UK: Where are we now? Nursing Times Research 8 (4) 293-307.

Goodyear, P., C. Jones, M. Asensio, V. Hodgson, and C. Steeples. 2005. Networked learning in higher education: students' expectations and experiences. Higher Education 50 473-508.

Gregory, M. 1995. Implications of the introduction of the Doctor of Education Degree in British universities: can the EdD reach parts the PhD cannot? The Vocational Aspect of Education 47 (2) 177-188.

HEFCE 2007. PhD research degrees: update: entry and completion. Higher Education Funding Council for England.

Halse, C., and J. Malfroy. 2010. Retheorising doctoral supervision as professional work. Studies in Higher Education 35 (1) 79-92.

Jaques, D., and G. Salmon. 2007. Learning in groups: a handbook for face-to-face and online environments. $4^{\text {th }}$ ed. Abingdon, Oxon: Routledge.

Jiang, L.C., N.N. Bazarova, and J.T. Hancock. 2011. The disclosure-intimacy link in computermediated communication: an attributional extension of the hyperpersonal model. Human Communication Research 37 58-77.

Lee, A. 2008. How are doctoral students supervised? Concepts of doctoral research supervision. Studies in Higher Education 33 (3) 267-281.

Leitch Review 2006. Prosperity for all in the global economy: world class skills. HMSO.

Linton, J.M., and S.M. Hedstrom. 2006. An exploratory qualitative investigation of group processes in group supervision: perceptions of Masters-level practicum students. The Journal for Specialists in Group Work 31 (1) 51-72.

Lyotard, J-F. 1984. The postmodern condition: a report on knowledge. Manchester: Manchester University Press.

McConnell, D. 2000. Implementing computer supported cooperative learning. $2^{\text {nd }}$ ed. London: Kogan Page.

Malfroy, J. 2005. Doctoral supervision, workplace research and changing pedagogic practices. Higher Education Research \& Development 24 (2) 165-178.

Samara, A. 2006. Group supervision in graduate education: a process of supervision skill development and text improvement. Higher Education Research \& Development 25 (2) 115-129.

Scott, D., A. Brown, I. Lunt, and L. Thorne. 2004. Professional doctorates: integrating professional and academic knowledge. Maidenhead: Open University Press.

Steeples, C., and C. Jones. (eds.) 2002. Networked learning: perspectives and issues. London: Springer.

Reference this paper as: Hutchings, M. (2015). Improving doctoral support through group supervision: analyzing face-to-face and technology-mediated strategies for nurturing and sustaining scholarship. Studies in Higher Education, 2015 http://dx.doi.org/10.1080/03075079.2015.1058352 
Taylor, S., and N. Beasley. 2005. A handbook for doctoral supervisors. Abingdon, Oxon: Routledge. Tidwell, L.C., and J.B. Walther. 2002. Computer-mediated communication effects on disclosure, impressions, and interpersonal evaluations: getting to know one another a bit at a time. Human Communication Research 28 (3) 317-348.

UKCGE (United Kingdom Council for Graduate Education). 2002. Report on professional doctorates. Dudley: UKCGE.

Usher, R. 2002. A diversity of doctorates: fitness for the knowledge economy? Higher Education Research \& Development 21 (2) 143-153.

Walther, J. B. 1996. Computer-mediated communication: impersonal, interpersonal, and hyperpersonal interaction. Communication Research 23 (1) 3-43.

Warry Report. 2006. Increasing the economic impact of Research Councils. Research Council: Economic Impact Group.

Wisker, G., G. Robinson, and M. Shacham. 2007. Postgraduate research success: communities of practice involving cohorts, guardian supervisors and online communities. Innovations in Education and Teaching International 44 (3) 301-320. 